\title{
Braquiterapia ocular en el tratamiento del retinoblastoma. Experiencia en el Hospital Infantil de México
}

\section{Ocular brachytherapy in the treatment of retinoblastoma. Experience in the Hospital Infantil de México}

\author{
Lorena Ramírez-Patiño ${ }^{1}$, Margarita Barnoya-Pérez de Engel ${ }^{2}$, Norma C. Lara-Molina', \\ Heynar Pérez-Villanueva ${ }^{3}$ y Marco A. Ramírez-Ortiz ${ }^{1 *}$
}

${ }^{1}$ Servicio de Oftalmología, Hospital Infantil de México Federico Gómez, Ciudad de México, México; ${ }^{2}$ Servicio de Oncología Ocular, Hospital Herrera Llerandi, Guatemala, Guatemala; ${ }^{3}$ Servicio de Radioterapia, Hospital Infantil de México Federico Gómez, Ciudad de México, México

\section{Resumen}

Objetivo: Describir los resultados del tratamiento con braquiterapia ocular en ojos únicos en pacientes con retinoblastoma (Rb) bilateral, así como las complicaciones de su uso como tratamiento primario o secundario. Métodos: Serie de casos donde se identificaron todos los pacientes con Rb en el Hospital Infantil de México tratados con braquiterapia ocular con yodo-125 como salvamento ocular después del fallo en el control de la neoplasia mediante quimioterapia intravenosa, tratamiento focal con láser o crioterapia y radioterapia externa. Resultados: Se incluyeron un total de siete pacientes, dos pacientes eran del sexo femenino, y cinco, de sexo masculino. Dos pacientes tenían Rb hereditario y seis eran bilaterales. Tres de los ojos tratados con braquiterapia eran derechos. El seguimiento promedio de estos pacientes fue de 5.35 años (rango 3.3 a 11 años). El éxito del tratamiento se alcanzó en cinco de los siete pacientes. Dos pacientes fueron enucleados a pesar del tratamiento con braquiterapia por falla en el control de la neoplasia. Uno de los pacientes falleció por neoplasia secundaria. Conclusiones: El tratamiento con braquiterapia ocular en un hospital de tercer nivel ofrece posibilidades de preservación ocular en pacientes con Rb bilateral y tratamiento oncológico máximo.

Palabras clave: Retinoblastoma. Braquiterapia. Cáncer. Ceguera adquirida. Terapia de salvamento.

\begin{abstract}
Objective: To describe results and complications of salvage eye treatment with brachytherapy in patients with diagnosis of bilateral retinoblastoma $(R b)$ and only one eye. Methods: Case series where we included all retinoblastoma patients treated with ocular brachytherapy with lodine-125 at the Hospital Infantil de Mexico after failure of eye salvage with intravenous chemotherapy, focal transpupillary laser o transconjunctival cryotherapy and external beam radiotherapy. Results: We analyzed 7 patients, 2 females and 5 males. Two had hereditary retinoblastoma and all had bilateral disease. Mean follow-up after treatment was 5.35 years (range 3.3-11 years). Tumor control was achieved in five of seven patients; two patients undergone enucleation due to failure to achieve tumor control. One of the patients died because of secondary neoplasm.
\end{abstract}

\section{Correspondencia:}

*Marco Antonio Ramírez Ortiz

Dr. Marquez, 162

Col. Doctores, Del. Cuauhtémoc

Fecha de recepción: 18-12-2018

C.P. 06720, Ciudad de México, México

E-mail: marco@unam.mx

0187-4519/@ 2019 Sociedad Mexicana de Oftalmología. Publicado por Permanyer México. Este es un artículo Open Access bajo la licencia CC BY-NC-ND (http://creativecommons.org/licenses/by-nc-nd/4.0/).

Disponible en internet: 01-07-2019 Rev Mex Oftalmol. 2019;93(4):194-199 www.rmo.com.mx 
Conclusions: Ocular brachytherapy treatment in a tertiary care center offers possibilities of ocular preservation in patients with bilateral $R b$ and maximum oncological treatment.

Key words: Retinoblastoma. Brachytherapy. Cancer. Acquired blindness. Salvage therapy.

\section{Introducción}

El salvamento ocular en el retinoblastoma $(R b)$ intraocular es un proceso que implica la utilización de diversos tipos de tratamiento, locales y sistémicos, para evitar la enucleación. Generalmente está indicado realizar salvamento ocular en el ojo menos afectado en aquellos pacientes con $\mathrm{Rb}$ bilateral, o en aquellos casos con tumores confinados a la retina y que no hayan desprendido la retina más de $3 \mathrm{~mm}$ alrededor de la lesión y no estén acompañados de siembras vítreas difusas. Existen varias opciones terapéuticas para controlar los tumores intraoculares. Los medios focales más utilizados son la crioterapia ${ }^{1}$, la fotocoagulación directa del tumor ${ }^{2,3}$, la termoterapia ${ }^{4}$, la radioterapia externa ${ }^{5,6} \mathrm{y}$, más recientemente, las inyecciones intravítreas de quimioterapia ${ }^{7}$ y la quimioterapia supraselectiva intaraarteria| $\left.\right|^{8,9}$. Todos estos tratamientos tienen complicaciones y secuelas y su uso está limitado a tumores intraoculares. La radioterapia externa es otra opción terapéutica y está indicada cuando el tratamiento focal no es suficiente para detener la progresión del tumor. Sin embargo, está asociada a un incremento en el riesgo de desarrollo de nuevas neoplasias diferentes al $\mathrm{Rb}^{10}$, pues el área del campo de tratamiento $\mathrm{y}$, en menor medida, los tejidos de las demás partes del cuerpo se ven expuestos a los efectos de la radiación, que produce cambios en el ADN de pacientes que podrían tener alterado su genoma ${ }^{11}$. La radioterapia focal o braquiterapia es un método que ha demostrado eficacia en el control de tumores intraoculares sin incrementar el riesgo de segundas neoplasias ${ }^{12}$. Asimismo, la braquiterapia se ha utilizado con éxito en el control de pacientes con Rb previamente tratados con quimioterapia intraarterial ${ }^{13}$, por lo que representa una opción viable en el tratamiento del $\mathrm{Rb}$. En este trabajo reportamos la experiencia en el tratamiento del $\mathrm{Rb}$ con braquiterapia del Servicio de Oftalmología del Hospital Infantil de México en conjunto con el Instituto de Cáncer de Guatemala.

\section{Pacientes y métodos}

Se incluyeron aquellos pacientes con $\mathrm{Rb}$ diagnosticados en el Hospital Infantil de México y que fueron tratados con braquiterapia ocular y que contaran al menos con 1 año de seguimiento. Todos los pacientes fueron tratados inicialmente en nuestro hospital y fueron agrupados de acuerdo con la Clasificación Internacional de $\mathrm{Rb}^{14}$.

Los tratamientos previos a la braquiterapia fueron individualizados. Cada uno de los pacientes recibió tratamiento sistémico a base de quimioterapia en doble o triple esquema a consideración del médico oncólogo pediatra. El tipo de tratamiento focal y la cantidad administrada fue determinada por el médico oftalmólogo pediatra dependiendo de la localización, tamaño del tumor y presencia o no de siembras vítreas. La cantidad de aplicaciones de láser, crioterapia, ciclos de quimioterapia y radioterapia externa que cada paciente recibió se describen en la Tabla 1.

La indicación del tratamiento con braquiterapia se realizó en aquellos pacientes para un único ojo cuyo tratamiento con fotocoagulación con láser diodo $810 \mathrm{~nm}$, crioterapia transconjuntival, quimioterapia intravenosa e incluso radioterapia externa no hubiera sido suficiente para controlar el tumor. Se hizo siguiendo los estándares internacionales: tumores ecuatoriales o periféricos cuyo diámetro sea menor de $15 \mathrm{~mm}$ en su base y no más de $10 \mathrm{~mm}$ de altura ${ }^{15,16}$. El tratamiento se realizó en la Ciudad de Guatemala por uno de los autores (MBP), con asistencia de uno de los coautores (NCLM). A todos los pacientes se les realizó un examen oftalmológico completo que incluyó: toma de agudeza visual, oftalmoscopia binocular, ultrasonografía ocular y fotografías de retina con RetCam III (Clarity Medical Systems, Pleasanton, Calif). Asimismo, todos los pacientes tuvieron un seguimiento oncológico completo que incluyó las revisiones clínicas y la realización de una resonancia magnética nuclear de órbitas y cráneo para identificar recidivas intraoculares, orbitarias e intracraneales.

Los siete pacientes de nuestra serie viajaron a Guatemala para la colocación de la placa de braquiterapia con recursos propios y obtenidos de donaciones de diversas fundaciones para el cáncer infantil. El seguimiento en todos los casos se realizó en el Hospital Infantil de México.

Todos los pacientes usaron placas circulares de $16 \mathrm{~mm}$ de diámetro tipo Bebig (Eckert \& Ziegler, Berlín, Alemania), con cubierta de oro con 13 semillas de yodo125, con una actividad mediana de 1.858 milicurios por semilla. Se irradiaron durante 90 horas con una dosis total máxima de 89.9 Grays, calculadas previamente en 
Tabla 1. Características clínicas de pacientes con retinoblastoma bilateral tratados con braquiterapia ocular

\begin{tabular}{|c|c|c|c|c|c|c|c|}
\hline Paciente & 1 & 2 & 3 & 4 & 5 & 6 & 7 \\
\hline Sexo & $\mathrm{F}$ & $\mathrm{F}$ & M & M & M & M & M \\
\hline Historia familiar & No & No & No & No & Sí & No & Sí \\
\hline Edad al diagnóstico (meses) & 15 & 22 & 14 & 35 & 2 & 20 & 21 \\
\hline Edad al momento de braquiterapi (meses) & 76 & 120 & 56 & 64 & 68 & 36 & 37 \\
\hline Aplicaciones láser & 7 & 4 & 8 & 8 & 10 & 14 & 10 \\
\hline Crioterapia & 2 & 7 & 0 & 4 & 3 & 0 & 6 \\
\hline Ciclos de quimioterapia & 8 & 7 & 15 & 6 & 8 & 16 & 10 \\
\hline Radioterapia externa & 1 & 2 & No & 1 & 1 & 1 & 1 \\
\hline Agudeza visual inicial & $20 / 30$ & $20 / 40$ & $20 / 32$ & $20 / 60$ & $20 / 400$ & $20 / 80$ & $20 / 100$ \\
\hline Agudeza visual final & $20 / 40$ & $20 / 80$ & $20 / 32$ & Enucleación & $20 / 400$ & $20 / 200$ & Enucleación \\
\hline Neoplasias secundarias & No & No & No & No & No & No & Sí \\
\hline Seguimiento después de la braquiterapia (meses) & 134 & 76 & 54 & 53 & 42 & 53 & 39 \\
\hline
\end{tabular}

función de la masa tumoral. Las semillas de yodo-125 fueron adheridas a la superficie interna de la placa de acuerdo a la planeación realizada por el radioterapeuta y previo al procedimiento quirúrgico. Al cumplir con la dosis calculada, se retiró el implante de yodo-125, con un procedimiento similar al de implantación.

La técnica quirúrgica fue la siguiente: bajo anestesia general previa antisepsia de la región periocular con colocación de campos estériles y blefaróstato, se realiza disección de la conjuntiva sobre la zona planeada para la colocación de la placa episcleral; se realiza oftalmoscopia indirecta con técnica estéril e indentación escleral para identificar con precisión la ubicación de la base tumoral; se marca con tinta indeleble la zona planeada identificada; en caso de que el tumor se encontrara por debajo de algún músculo extraocular, se desinsertó para el adecuado amoldamiento de la placa a la curvatura ocular y colocación precisa en la base del tumor; posteriormente se procedió a suturar la placa a la esclera con Vicryl 6-0 utilizando los orificios diseñados para su soporte; se aseguró de que existiera un mínimo de movimiento para garantizar la emisión de la radiación en la zona planeada y disminuir la disipación de energía en zonas retinianas sanas; se realizó hemostasia por compresión con gasa e hisopos; la cápsula de Tenon y la conjuntiva fueron suturadas con puntos simples separados con Vicryl 6-0; se mantuvo la placa el tiempo calculado para la emisión de radiación (en promedio $72 \mathrm{~h}$ ); una vez transcurrido el periodo planeado de radiación, se realizó el retiro de la placa en un nuevo procedimiento quirúrgico, bajo anestesia general y técnica estéril, y, finalmente, se reinsertó músculo extraocular en su sitio original si este fue desinsertado.

Se definió el tratamiento como un éxito al obtener control e inactividad completa del tumor durante un año después a la fecha de inicio del tratamiento con braquiterapia. Estos criterios de inactividad se definieron mediante patrones de regresión tumoral descritos por Singh $^{17}$ : tipo 0 , el tumor desaparece completamente sin dejar cicatriz; tipo I, masa completamente calcificada (queso cottage); tipo II, tumor residual translúcido de color grisáceo con apariencia en "carne de pescado»; tipo III, combinación de regresiones tipo I y tipo II, y tipo IV, cicatriz coriorretiniana atrófica plana.

Los procedimientos utilizados en todos nuestros pacientes se realizaron con la autorización del Comité de Ética de ambos hospitales y con el consentimiento informado por parte de los padres de los pacientes.

Se utilizó estadística descriptiva para la evaluación los resultados de este trabajo mediante el software Excel.

\section{Resultados}

Durante el periodo de 1997 a diciembre del 2016, de una base de datos de 406 pacientes con $\mathrm{Rb}$ fueron identificados siete pacientes con enfermedad bilateral que recibieron tratamiento con braquiterapia epiescleral para salvamento del ojo menos afectado. La edad promedio al momento de la braquiterapia era de 5.3 años 
(rango: 3 a 10 años). La indicación del tratamiento con braquiterapia ocular se decidió por falla en el control del tumor a pesar del tratamiento focal y sistémico. Dos pacientes eran del sexo femenino. Una cuarta parte de los pacientes tenían $\mathrm{Rb}$ familiar. Todos los pacientes presentaban antecedente de quimioterapia previa, con una combinación de carboplatino, etopósido y ciclofosfamida. El tiempo promedio desde el diagnóstico del $\mathrm{Rb}$ a la colocación de la placa fue de 3.8 años (rango 1.3 a 8 años). El seguimiento promedio de estos pacientes fue de 5.35 años (rango 3.3 a 11 años). En la Tabla 1 podemos observar las características generales de los pacientes estudiados.

En cuanto a las características de los tumores tratados, en la Tabla 2 podemos ver las medidas, localizaciones, presencia o ausencia de siembras vítreas, así como las fotografías clínicas preoperatorias y en su último seguimiento de cada uno de los siete pacientes incluidos en este estudio.

El éxito del tratamiento se alcanzó en cinco de los siete pacientes (71.4\%). En dos pacientes no se logró controlar el tumor, por lo que tuvieron que ser sometidos a enucleación; uno de ellos falleció por neoplasia secundaria en los senos paranasales.

En cuanto a las complicaciones, cinco pacientes las desarrollaron y todos ellos tenían tratamiento previo con radioterapia externa. La más frecuente fue la catarata posradiación (28.6\%), en tanto que la hemorragia posbraquiterapia, la maculopatía posradiación y el ojo seco grave se presentaron en un caso cada uno, con una frecuencia del $14.3 \%$. Los dos pacientes que desarrollaron catarata por radiación fueron tratados con facoemulsificación más implante de lente intraocular, y presentaron una agudeza visual final de 20/40 y 20/200, respectivamente. El caso del paciente que presentó la hemorragia posterior a la braquiterapia se resolvió con tratamiento conservador, y obtuvo una agudeza visual final de 20/32. La maculopatía por radiación se trató de forma exitosa con tres aplicaciones de terapia antiangiogénica intravítrea (bevacizumab). Actualmente, esta paciente tiene una agudeza visual de 20/80. El paciente que desarrolló ojo seco grave no respondió al reemplazo con lágrimas artificiales y tapón lagrimal, desarrolló opacidad corneal parcial y vascularización corneal superficial. Su agudeza visual final fue de 20/400. En la Tabla 3 se describen las complicaciones desarrolladas en nuestra serie.

\section{Discusión}

La braquiterapia es una modalidad de tratamiento del cáncer intraocular que se ha utilizado para el

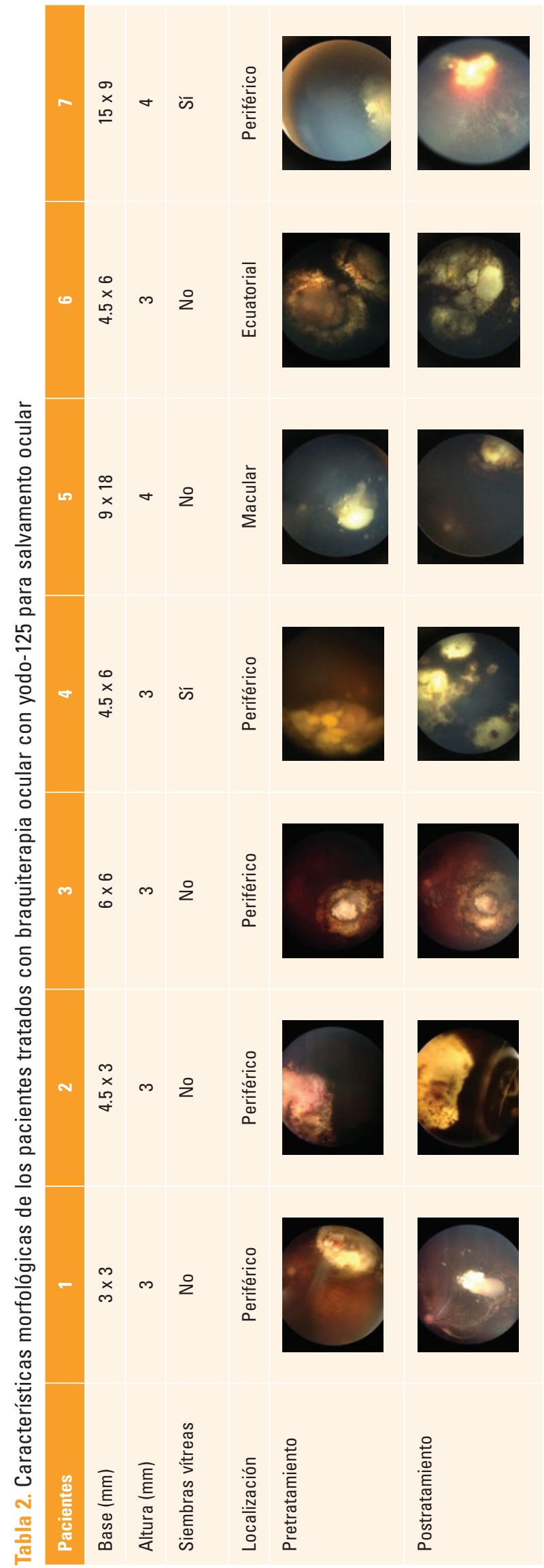


Tabla 3. Complicaciones oculares en pacientes tratados con braquiterapia ocular con yodo-125 para salvamento ocular

\begin{tabular}{|l|l|}
\hline Complicación & N $(\%)$ \\
\hline Catarata & $2(28.6)$ \\
\hline Hemorragia retiniana & $1(14.3)$ \\
\hline Maculopatía por radiación & $1(14.3)$ \\
\hline Ojo seco grave & $1(14.3)$ \\
\hline
\end{tabular}

tratamiento del $\mathrm{Rb}$ desde hace casi un siglo ${ }^{18}$. Su uso ha sido extenso en los centros de referencia más importantes a nivel mundial y se han publicado diferentes reportes sobre su eficacia ${ }^{19-21}$. La mayor parte de los centros de referencia mundiales utilizan el yodo-125, sin embargo, el rutenio también ha demostrado eficiencia en el control de esta neoplasia ${ }^{22}$.

Como tratamiento primario, la placa de radioterapia ofrece hasta un $88 \%$ de éxito en tumores no mayores a $15 \mathrm{~mm}$ de base y $9 \mathrm{~mm}$ de grosor y sin o con mínimas siembras vítreas ${ }^{21}$. En caso de tratamiento secundario usado en tumores recurrentes o con falla a otros tratamientos, como los pacientes de esta serie, la placa de radioterapia ofrece un control tumoral del $92 \%$ después del tratamiento focal con láser o crioterapia, del $92 \%$ después de quimiorreducción, del $75 \%$ después de radioterapia externa y del $66 \%$ después de quimiorreducción y radioterapia externa ${ }^{20}$. En nuestra serie, tuvimos un salvamento ocular del $71.4 \%$, ya que dos ojos tratados no respondieron al tratamiento y tuvieron que ser enucleados. Asimismo, la efectividad de la braquiterapia se ha reportado que no decrece a pesar de tratamientos focales previos ${ }^{23}$.

La Guía de la Sociedad Americana de Braquiterapia para el tratamiento del $\mathrm{Rb}$ recomienda el uso de este método como tratamiento secundario después de la falla del tratamiento focal y sistémico ${ }^{15}$. También señalan que los tumores ideales para esta forma de tratamiento son los de localización anterior al ecuador. En nuestra serie, cinco de los siete pacientes cumplieron son esta característica.

En una serie de 208 casos, Shields, et al. reportaron en los pacientes con tratamiento secundario las siguientes complicaciones: catarata $(31 \%)$, con retinopatía proliferativa (20\%), papilopatía (21\%), maculopatía no proliferativa (12\%), proliferativa (10\%) y glaucoma $(7 \%)^{21}$. En nuestra serie, la catarata fue la complicación más frecuente, pues estuvo presente en todos los pacientes con antecedente de radioterapia externa, seguida por la maculopatía no proliferativa, hemorragia vítrea y ojo seco grave. La aparición de maculopatía por radiación se ha reportado en pacientes incluso después de 40 años de tratamiento ${ }^{24}$, por lo que es posible que esta complicación aparezca en más pacientes de nuestra serie con un seguimiento más prolongado.

Los tumores que mejor respondieron al tratamiento fueron los que presentaban tumores de localización periférica, de menor tamaño (base y altura menor a $6 \mathrm{~mm}$ ) y que no tuvieran siembras vítreas; siendo el caso 1,2 y 3 los de mejor agudeza visual final, con 20/40, 20/80 y 20/30, respectivamente.

En nuestra serie tuvimos dos casos de fallo al tratamiento del $\mathrm{Rb}$ con el uso de radioterapia focal. En el tiempo de seguimiento se realizaron dos enucleaciones en los ojos tratados previamente con este método: el primer paciente, a los 2 meses de recibir la braquiterapia, ya que el tumor y las siembras vítreas progresaron a pesar del tratamiento. El segundo paciente fue enucleado 1 año y 8 meses después de la braquiterapia por falta de control en la actividad del tumor a pesar de los múltiples tratamientos focales y sistémicos. EI paciente falleció por segunda neoplasia 3 años y 10 meses después de la braquiterapia.

A pesar de los beneficios de este tratamiento, en México existe un acceso muy limitado a tratamientos con braquiterapia para distintos tumores intraoculares en adultos y aún no hay reportes de sus resultados por el poco tiempo que lleva utilizándose. En hospitales pediátricos públicos en nuestro país, no se cuenta actualmente con los recursos económicos y de infraestructura suficientes para implementar la braquiterapia como un tratamiento de rutina en los pacientes con $\mathrm{Rb}$. La incorporación de esta modalidad de tratamiento a hospitales de referencia nacionales ofrecerá alternativas adicionales a pacientes con $\mathrm{Rb}$ de difícil control, que incluso no han podido ser resueltas con quimioterapia intraarterial supraselectiva ${ }^{13}$.

En conclusión, la aplicación de braquiterapia ocular en un hospital de tercer nivel ofrece posibilidades de preservación ocular en ciertos pacientes con tratamiento oncológico máximo fallido y buena agudeza visual. El salvamento ocular en pacientes con $\mathrm{Rb}$ es un proceso muy largo, complejo y costoso. Además, requiere de la intervención de un equipo médico multidisciplinario y de diferentes opciones de tratamiento que permitan evitar la pérdida del globo ocular sin poner en riesgo la vida del paciente.

\section{Conflicto de intereses}

Los autores declaran que no existe conflicto de intereses. 


\section{Responsabilidades éticas}

Protección de personas y animales. Los autores declaran que para esta investigación no se han realizado experimentos en seres humanos ni en animales.

Confidencialidad de los datos. Los autores declaran que han seguido los protocolos de su centro de trabajo sobre la publicación de datos de pacientes.

Derecho a la privacidad y consentimiento informado. Los autores han obtenido el consentimiento informado de los pacientes y/o sujetos referidos en el artículo. Este documento obra en poder del autor de correspondencia.

\section{Bibliografía}

1. Abramson DH, Ellsworth RM, Rozakis GW. Cryotherapy for retinoblastoma. Arch Ophthalmol. 1982;100:1253-6.

2. Shields JA, Shields CL, Parsons H, Giblin ME. The role of photocoagulation in the management of retinoblastoma. Arch Ophthalmol. 1990;108:205-8.

3. Murphree AL, Villablanca JG, Deegan WF, $3^{\text {rd }}$, Sato JK, Malogolowin M, Fisher A, et al. Chemotherapy plus local treatment in the management of intraocular retinoblastoma. Arch Ophthalmol. 1996;114:1348-56.

4. Shields CL, Santos MC, Diniz W, Gunduz K, Mercado G, Cater JR, et al. Thermotherapy for retinoblastoma. Arch Ophthalmol.1999;117:885-93.

5. Toma NM, Hungerford JL, Plowman PN, Kingston JE, Doughty D. External beam radiotherapy for retinoblastoma: II. Lens sparing technique. $\mathrm{Br}$ J Ophthalmol. 1995;79:112-7.

6. Munier FL, Verwey J, Pica A, Balmer A, Zografos L, Abouzeid H, et al. New developments in external beam radiotherapy for retinoblastoma: from lens to normal tissue-sparing techniques. Clin Experiment Ophthalmol. 2008:36:78-89

7. Munier FL, Gaillard MC, Balmer A, Soliman S, Podilsky G, Moulin AP, Beck-Popovic M. Intravitreal chemotherapy for vitreous disease in retinoblastoma revisited: from prohibition to conditional indications. $\mathrm{Br} \mathrm{J} O \mathrm{Oph}-$ thalmol. 2012;96:1078-83.

8. Abramson DH, Dunkel IJ, Brodie SE, Kim JW, Gobin YP. A phase I/II study of direct intraarterial (ophthalmic artery) chemotherapy with melphalan for intraocular retinoblastoma initial results. Ophthalmology. 2008;115:1398-404.
9. Abramson DH, Marr BP, Brodie SE, Dunkel I, Palioura S, Gobin YP. Ophthalmic artery chemosurgery for less advanced intraocular retinoblastoma: Five year review. PLoS ONE. 2012;7:e34120.

10. Penn I. Second neoplasms following radiotherapy or chemotherapy for cancer. Am J Clin Oncol.1982;5:83-96.

11. Abramson DH, Frank CM. Second nonocular tumors in survivors of bilateral retinoblastoma: a possible age effect on radiation-related risk. Ophthalmology. 1998;105:573-9.

12. Merchant TE, Gould CJ, Wilson MW, Hilton NE, Rodriguez-Galindo C, Haik BG. Episcleral plaque brachytherapy for retinoblastoma. Pediatr Blood Cancer. 2004;43:134.9.

13. Francis JH, Barker CA, Wolden SL, McCormick B, Segal K, Cohen G, et al. Salvage/adjuvant brachytherapy after ophthalmic artery chemosurgery for intraocular retinoblastoma. Int J Radiat Oncol Biol Phys. 2013; 87:517-23.

14. Murphree AL. Intraocular retinoblastoma: the case for a new group classification. Ophthalmol Clin North Am. 2005;18:41-53.

15. American Brachytherapy Society - Ophthalmic Oncology Task Force. The American Brachytherapy Society consensus guidelines for plaque brachytherapy of uveal melanoma and retinoblastoma. Brachytherapy. 2014;13:1-14.

16. Shields JA, Shields CL, De Potter P, Hernandez JC, Brady LW. Plaque radiotherapy for residual or recurrent retinoblastoma in 91 cases. $\mathrm{J} \mathrm{Pe}$ diatr Ophthalmol Strabismus. 1994;31:242-5.

17. Singh AD, Garway-Heath D, Love S, Plowman PN, Kingston JE, Hungerford JL. Relationship of regression pattern to recurrence in retinoblastoma. Br J Ophthalmol. 1993;77:12-6.

18. Moore RF, Stallard HB, Milner JG. Retinal Gliomata Treated by Radon Seeds. Br J Ophthalmol. 1931;15:673-96.

19. Stallard HB. The conservative treatment of retinoblastoma. Trans Ophthalmol Soc U K. 1962;82:473-534.

20. Shields CL, Mashayekhi A, Sun H, Uysal Y, Friere J, Komarnicky L, et al. lodine 125 plaque radiotherapy as salvage treatment for retinoblastoma recurrence after chemoreduction in 84 tumors. Ophthalmology. 2006;113:2087-92.

21. Shields CL, Shields JA, Cater J, Othmane I, Singh AD, Micaily B. Plaque radiotherapy for retinoblastoma: long term tumor control and treatment complications in 208 tumors. Ophthalmology. 2001;108:2116-21.

22. Murakami N, Suzuki S, Ito Y, Yoshimura R, Inaba K, Kuroda Y, et al.106 Ruthenium plaque therapy (RPT) for retinoblastoma. Int J Radiat Oncol Biol Phys. 2012;84:59-65.

23. Lucas JT, McGee R, Billups CA, Qaddoumi I, Merchant TE, Brennan RC, et al. Prior non-irradiative focal therapies do not compromise the efficacy of delayed episcleral plaque brachytherapy in retinoblastoma. $\mathrm{Br} \mathrm{J}$ Ophthalmol. 2018 Jun 28. pii: bjophthalmol-2018-311923. doi: 10.1136/bjophthalmol-2018-311923

24. Bellerive C, Singh AD. Radiation Retinopathy 47 Years following Brachytherapy for Retinoblastoma. Ocul Oncol Pathol. 2018;4:157-60. 